\title{
Latest Results on Complex Plasmas with the PK-3 Plus Laboratory on board the International Space Station
}

\author{
M. Schwabe - C.-R. Du · P. Huber · A. M. Lipaev · V. I. Molotkov · V. N. Naumkin \\ S. K. Zhdanov · D. I. Zhukhovitskii · V. E. Fortov · H. M. Thomas
}

Received: date / Accepted: date

Abstract Complex plasmas are low temperature plasmas that contain microparticles in addition to ions, electrons, and neutral particles. The microparticles acquire high charges, interact with each other and can be considered as model particles for effects in classical condensed matter systems, such as crystallization and fluid dynamics. In contrast to atoms in ordinary systems, their movement can be traced on the most basic level, that of individual particles. In order to avoid disturbances caused by gravity, experiments on complex plasmas are often performed under microgravity conditions. The PK-3 Plus Laboratory was operated on board the International Space Station from 2006 - 2013. Its heart consisted of a capacitively coupled radio-frequency plasma chamber. Microparticles were inserted into the low-temperature plasma, forming large, homogeneous complex plasma clouds. Here, we review the results obtained with recent analyses of PK-3 Plus data: We study the formation of crystallization fronts, as well as the microparticle motion in, and structure of crystalline complex plasmas. We investigate fluid effects such as wave transmission across an interface, and the development of the energy spectra during the onset of turbulent micropar-

The PK-3 Plus project was funded by the space agency of the Deutsches Zentrum für Luft- und Raumfahrt e.V. with funds from the Federal Ministry for Economy and Technology according to a resolution of the Deutscher Bundestag under grant number 50WM1203. A. M. Lipaev, V. N. Naumkin, and D. I. Zhukhovitskii are supported by the Russian Science Foundation Grant No. 14-12-01235.

M. Schwabe $\cdot$ P. Huber $\cdot$ S. K. Zhdanov $\cdot$ H. M. Thomas

Institut für Materialphsyik im Weltraum, Deutsches Zentrum für Luftund Raumfahrt (DLR), Münchener Str. 20, 82234 Weßling, Germany;

E-mail: mierk.schwabe@dlr.de

C.-R. Du

College of Science, Donghua University, 201620 Shanghai, PRC

A. M. Lipaev · V. I. Molotkov · V. N. Naumkin · D. I. Zhukhovitskii · V.E. Fortov

Joint Institute of High Temperatures, Russian Academy of Sciences, Izhorskaya 13, Bd. 2, 125412 Moscow, Russia

ticle movement. We explore how abnormal particles move through, and how macroscopic spheres interact with the microparticle cloud. These examples demonstrate the versatility of the PK-3 Plus Laboratory.

Keywords Dusty plasma - Turbulence - Interfaces · Waves · Cavitation $\cdot$ Crystallization

PACS 52.27.Lw $\cdot$ 52.35.Fp $\cdot 64.70 . \mathrm{dg} \cdot 47.27 . \mathrm{Cn}$

\section{Introduction}

Studies under microgravity conditions remove the ubiquitous stress that gravity poses on a system and allow experiments under more isotropic conditions, or on time scales not accessible under gravity conditions [1-4]. This is also true for complex or dusty plasmas which are low temperature plasmas that contain microparticles as additional component to neutrals, ions, and electrons [5]. The ions and electrons charge the microparticles to several thousands of elementary charges, and the microparticles interact strongly with each other. Due to their relatively large mass and size, they move slowly enough to make tracing the individual microparticles possible, enabling observations at the kinetic level.

Under gravity conditions, the microparticles are suspended near the sheath region of the plasma, where they are levitated by strong electric fields, which also lead to strong ion fluxes [6,7] and instabilities [8]. In contrast, under microgravity conditions, the microparticles are suspended in the plasma bulk, where their interactions are more isotropic $[9,10]$. Under those conditions, typically a central particlefree void forms, which is caused by a balance between the drag force of ions streaming towards the chamber walls and electrostatic confinement and interparticle pressure $[11,12]$. The void can be closed under suitable experimental conditions [13]. 


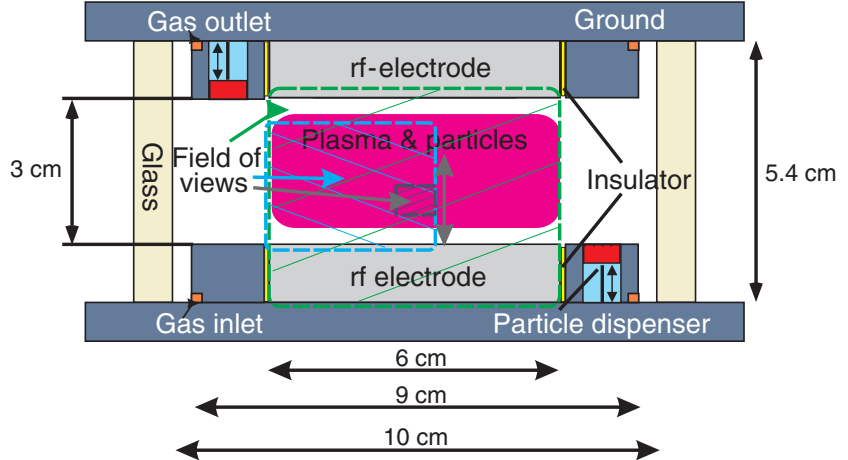

Fig. 1 (color online) Sketch of the plasma chamber of the PK-3 Plus Laboratory. The light green dashed line indicates the field of view of the overview camera, the blue dashed line that of the quadrant view camera, and the dark green dashed line that of the high resolution camera. The high resolution camera was moveable vertically, as is indicated with arrows. All mentioned cameras and the laser were mounted on a translation stage, which made scanning in horizontal direction possible. From [27]. OIOP Publishing \& Deutsche Physikalische Gesellschaft. Reproduced by permission of IOP Publishing. CC BY-NC-SA.

Weightless complex plasmas show a wealth of interesting phenomena such as relatively stress-free crystallization $[9,14]$, waves $[15,16]$, lane formation $[17,18]$, electrorheology and string formation [19-22], cavitation and Mach cones [23-25], etc. Laboratories to study complex plasmas were already used on board the Mir space station [26], and were among the first scientific experiments on board the International Space Station (ISS) [9]. The Russian-German PK-3 Plus Laboratory was operated on the ISS from 2006 - 2013 [27-29], and the state-of-the-art Russian-European PK-4 Laboratory is currently on board [30,31]. Data analysis from PK-3 Plus is still on-going, and here we will review some of the latest results.

Fig. 1 shows a sketch of the heart of the PK-3 Plus Laboratory, the plasma chamber. A plasma was produced in a vacuum chamber filled with argon or neon gas at pressures between 5 and $255 \mathrm{~Pa}$ by applying a radio-frequency electric field to two parallel electrodes with a distance of $3 \mathrm{~cm}$ and a radius of $6 \mathrm{~cm}$. The electrodes were surrounded by $1.5 \mathrm{~cm}$ wide grounded guard rings in which particle dispensers were embedded. The dispensers were shaken electromagnetically in order to inject monodisperse silica and melamine-formaldehyde (MF) particles with diameters between $1.55 \mu \mathrm{m}$ and $14.6 \mu \mathrm{m}$ through sieves into the plasma. Steel balls of $1 \mathrm{~mm}$ diameter inside the dispensers were used to break up any particle agglomerates. Tab. 1 gives the experimental conditions of the experiments presented here.

A laser sheet illuminated one vertical slice through the microparticle cloud. Three cameras were focused on this laser plane and recorded the motion of the microparticles. The fields of view of these cameras are shown in Fig. 1. In particular, the field of view of the high resolution cam-
Table 1 Conditions of the experiments reviewed in this paper (pressure $p$, effective voltage $U_{\text {eff }}$, microparticle diameter $d$ ). All experiments were done in argon. The particles with size $2.55 \mu \mathrm{m}$ and up were MF particles with a mass density of $1510 \mathrm{~kg} / \mathrm{m}^{3}$, the $1.55 \mu \mathrm{m}$ particles were composed of silica with a density of $1850 \mathrm{~kg} / \mathrm{m}^{3}$.

\begin{tabular}{lclll}
\hline Experiment & $p(\mathrm{~Pa})$ & $U_{\text {eff }}(\mathrm{V})$ & $\mathrm{d}(\mu \mathrm{m})$ & Ref. \\
\hline Crystals & $10-21$ & $13.2-14.6$ & $2.55-6.8$ & {$[34,33]$} \\
Turbulence & 9 & 13.1 & 9.2 & {$[35-37]$} \\
Waves & 10 & $\sim 21$ & $6.8 / 9.2$ & {$[38]$} \\
Photophoresis & $30-110$ & $8-14$ & $1.55-6.8$ & {$[39]$} \\
Spheres & 29 & 14.0 & $3.4 / 6.8$ & {$[40]$} \\
\hline
\end{tabular}

era was $8 \times 6 \mathrm{~mm}^{2}$ (shown in dark green in the figure), and that of the quadrant view camera (shown in blue) was $26 \times 36 \mathrm{~mm}^{2}$. The maximal frame rate was $50 \mathrm{fps}$. The laser and the high resolution and quadrant view cameras were mounted on a translation stage, which allowed scanning through ${ }_{37}$ the microparticle cloud in a tomographic procedure. The positions of the microparticles are identified in each experimental snapshot by finding pixel brightnesses above a suitable threshold and identifying the center of mass of the corresponding pixels, resulting in sub-pixel accuracy of the particle position [32,33]. The procedure can be adapted to obtain the three-dimensional particle position from scans [14, 34]. The particles can then be traced from frame to frame to study their dynamics or the static particle distribution.

\section{Plasma crystals}

The plasma sheath exerts strong forces on the microparticles, for instance via strong ion fluxes. This is beneficial to instabilities. Under microgravity conditions, the microparticles are levitated in the plasma bulk, where the sheath induced instabilities are not present. This leads to a different behavior than under gravity conditions. For instance, under gravity conditions, a crystalline microparticle cloud (called "plasma crystal" [41]) can be melted by an instability originating near the sheath $[42,43]$. When the plasma crystal is located further in the bulk of the plasma, the opposite behavior is typically observed - melting occurs at high pressures [44-46].

It is also possible to melt a plasma crystal by other methods, for instance, due to a drop in the particle charge [46], or by mechanical shaking. In PK-3 Plus, this was achieved by using a function generator to apply an additional electric fields to a plasma crystal composed of $1.55 \mu \mathrm{m}$ silica microparticles in an argon plasma at $10 \mathrm{~Pa}$. The electric field shook the plasma crystal, which lead to melting. The microparticle cloud then recrystallized in fronts. An example of these fronts can be seen in Fig. 2. The crystalline regions can be found by either superposing several experimental images to measure the amount by which the microparti- 


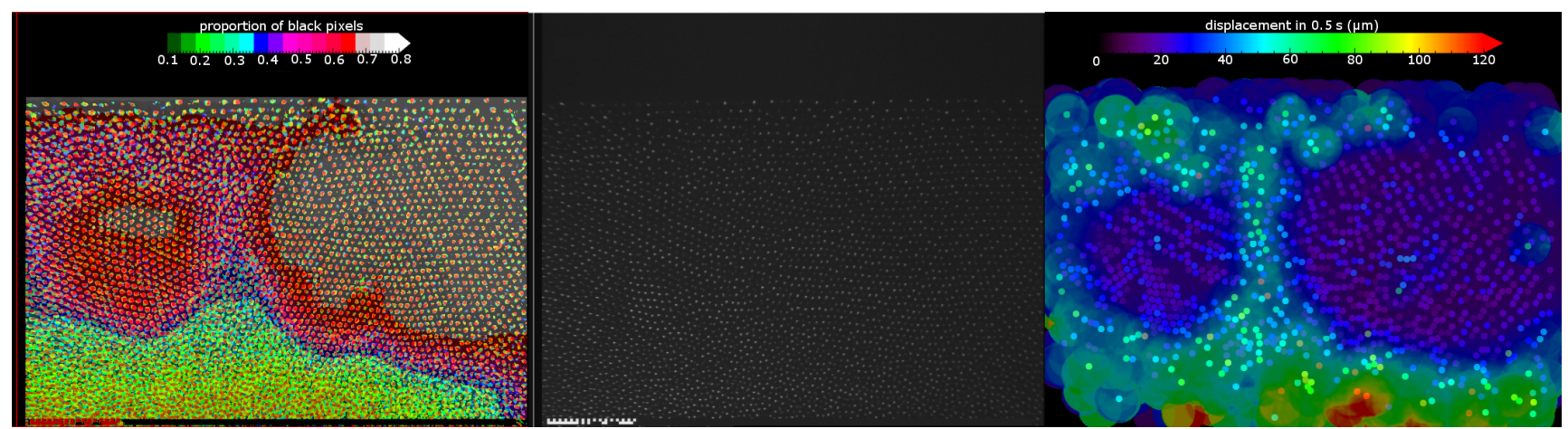

Fig. 2 (color online) View with the high resolution camera of crystallization fronts spreading in a microparticle cloud composed of $1.55 \mu \mathrm{m}$ particles in argon at $10 \mathrm{~Pa}$. Two crystalline regions are visible, a smaller one in the left and a larger one in the right. The two are separated by a liquid bridge. (left) Five consecutive experimental images overlaid, with color coding from blue to red. The background is colored according to the local amount of black pixels, which can be regarded a measure of movement of the microparticles [47]. (center) Original image. Field of view: $8 \times 6 \mathrm{~mm}^{2}$. (right) Detected particle positions. The background is color-coded according to the mean displacement of microparticles during $0.5 \mathrm{~s}$.

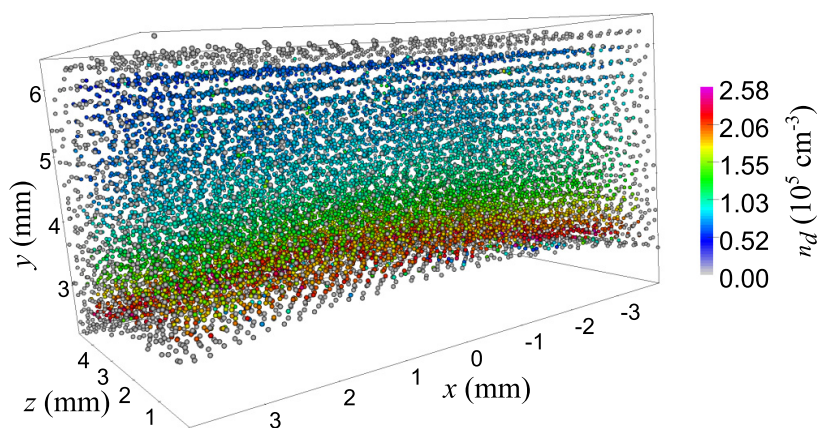

Fig. 3 (color online) Three-dimensional distribution of microparticles in a plasma crystal located above the void. The color indicates the local microparticle number density. Reprinted figure with permission from Naumkin et al., Phys. Rev. E 94, 033204 (2016). Copyright (2016) by the American Physical Society.

As can be seen in the image, two crystalline domains were present, which were separated by a liquid bridge. The details on these crystallization fronts will be reported in a future publication.

As the microparticle positions are rather stable, a plasma crystal is ideal to perform three-dimensional studies. For instance, the microparticle number density distribution can be studied in detail. For this purpose, the particle positions were recorded by the high resolution camera while short scans in horizontal (parallel to the electrodes) direction were performed at various experimental settings, see Tab. 1, row "Density" [34]. The local number density of microparticles was then calculated as the inverse volume of the 3D Voronoi cells. Fig. 3 shows the spatial distribution of microparticle positions and number density. It can be seen that the microparticle distribution is not completely isotropical and homogeneous: near the electrodes (in the top of the pic- ture), they form parallel planes, which is due to the chamber geometry; the planes form in direction perpendicular to the electric field. Also, the microparticle density increases sharply near the void edge (in the bottom of the picture). This is typical for microgravity experiments in capacitively coupled plasmas $[13,48]$. The formation of this high density region may be due to an inversion of the direction of the ion flow $[49,34]$. In the region further away from the void edge, the microparticle density changes less sharply. Naumkin et al. [34] found that in that region, for low pressures (around $10 \mathrm{~Pa}$ in argon), the density decreased monotonically with distance from the discharge center. At higher pressures, the density was almost constant. Based on the measured microparticle densities, the ion and electron densities were estimated to be around $10^{15} \mathrm{~m}^{-3}$ by fitting theoretical models to the measured microparticle density distribution [34].

Zhukhovitskii et al. [33] used data from the same experiments without scans to study the fluctuation of microparticle positions inside their cages in the plasma crystal. The magnitude of fluctuations increased with particle diameter and gas pressure, but were isotropic within the plasma crystal, almost homogeneous along the horizontal direction, and weakly inhomogeneous along the vertical direction. This corresponds to the distribution of the number density. The oscillations of individual microparticles were correlated with their nearest neighbors. The authors show that the microparticle kinetic temperature lay in the range of $(1-4) \mathrm{eV}$. This is two orders of magnitude larger than the gas temperature, and thus indicative of anomalous kinetic heating, which might be the result of large charge fluctuations within the plasma crystal. In contrast to previous experiments [50], the microparticle oscillations here were purely isotropic and thus could not have been caused by the ion flux or ambipolar electric field. 


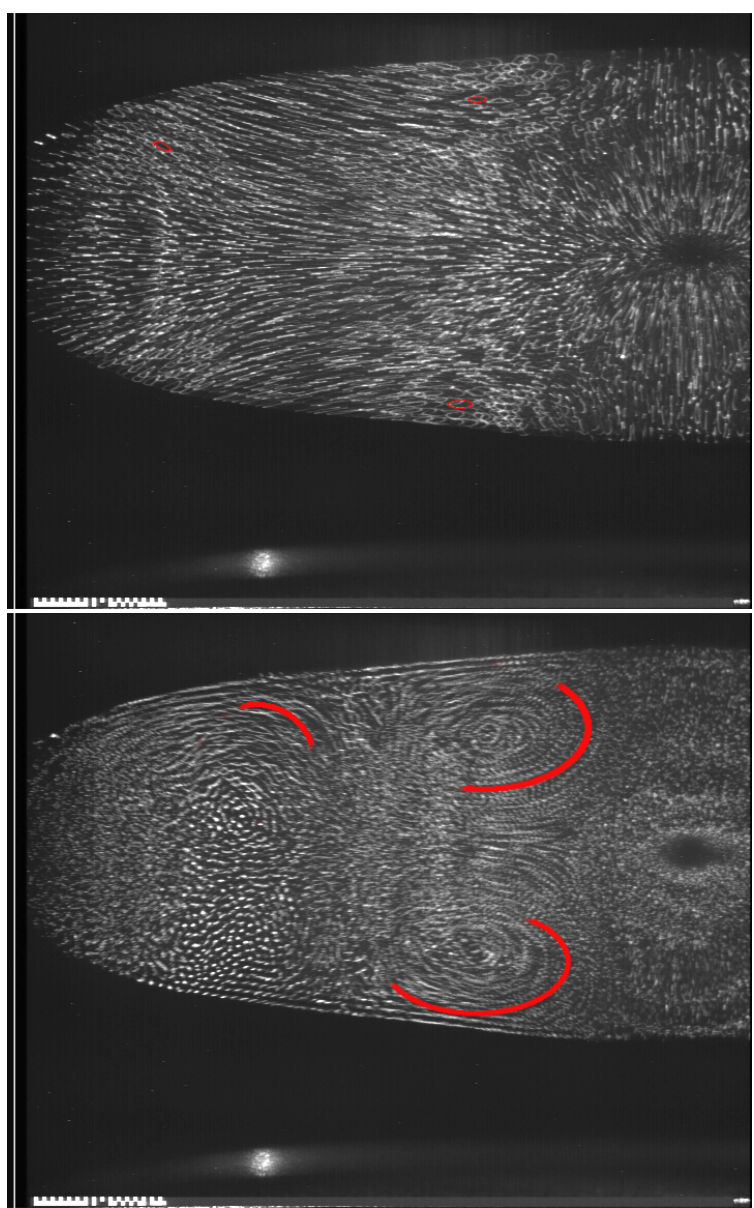

Fig. 4 (color online) (Top) All experimental snapshots from one heartbeat oscillation are superposed (17 images with time step of $0.02 \mathrm{~s}$, corresponding to a total time of $0.32 \mathrm{~s}$ ). A general movement of the particles in radial direction is well visible, as is the motion in small vortices. (Bottom) 17 experimental snapshots taken within the same phase of the heartbeat oscillation are superposed (time step $0.34 \mathrm{~s}$, total time covered $5.4 \mathrm{~s}$ ). Now intermediate and large scale vortices become visible. Field of view: $26 \times 36 \mathrm{~mm}^{2}$. The contrast of the images was enhanced. Some exemplary vortices are indicated in red.

\section{Turbulence}

The complex plasma is not always as stable as a plasma crystal. Under some conditions, instabilities can set in. A prominent example is the heartbeat instability $[51,52]$, in which the microparticle-free void oscillates radially, which leads to a repeated radial motion of the microparticles. The reason for the development of this instability might be the loss of electrons and ions on microparticles around the void and subsequent formation of a sheath [53]. The development of the heartbeat instability can be suppressed by applying an alternating electric field in the range of some tens of $\mathrm{Hz}$ (in the present experiment $(42-51) \mathrm{Hz}$ ). Once this field is switched off, the heartbeat develops.

This technique was applied in [35-37]. After the heartbeat developed, the microparticle motion became chaotic, which can be seen in Fig. 4. A general radial motion of the microparticles is overlaid with vortex movement on several spatial and time scales. Schwabe, Zhdanov and Räth [36,37] demonstrated that the kinetic energy spectrum $(E)$ before the instability onset can be described by an exponential dependence on the wave number $k$, and by a power law during the unstable phase. The power law splits into two regions with dependences $E \propto k^{-5 / 3}$ and $E \propto k^{-3}$. This dependence is also seen in the double cascade predicted for forced twodimensional turbulence by Kraichnan [54] and Leith [55]. The two parts of the spectrum meet at the wave number corresponding to the scale of the heartbeat, which is the source of energy for the microparticle motion. This discovery warrants further examination, and demonstrates the potential of complex plasmas to study turbulence on the level of the carriers of the turbulent interactions.

\section{Wave transmission across an interface}

Another mechanism by which a complex plasma cloud may become instable is via waves. Dust acoustic waves, that is, low-frequency density waves in the microparticle cloud, are typically excited by strong ion fluxes through the cloud when the gas pressure falls below a threshold [56-60]. Then, a two-stream instability develops, and waves start to propagate inside the microparticle cloud. Waves can also be triggered by the heartbeat instability $[52,61]$, or be externally excited with a function generator [62-65]. Within the waves, the microparticles typically move in the wave fronts with a velocity a little slower than that of the front, before leaving the front and falling back towards their original positions [66]. Trapping of microparticles inside the waves is also possible $[67,68]$. The movement of the microparticles can also be three-dimensional, with strong components in direction perpendicular to the direction of wave propagation [69].

With PK-3 Plus, the wave propagation across an interface formed between two clouds composed of microparticles of different sizes - a "binary" complex plasma - was recently observed by Yang et al. [38]. In capacitively coupled plasmas, the microparticles arrange by size, as the ratio of the ion drag force (accelerating the particles towards the chamber edges) and the electric force (confining the particles in the chamber) depends on the particle radius [17]. Another reason for a fluid phase separation in binary complex plasmas is the asymmetry in the interactions between the two particle types [70].

Fig. 5 shows an overlay of five experimental images recorded with the quadrant view camera in a binary complex plasma composed of $6.8 \mu \mathrm{m}$ and $9.2 \mu \mathrm{m}$ particles in argon at $10 \mathrm{~Pa}$. The smaller particles arrange around the void near the center of the plasma chamber, the larger particles closer to the edges. The gray line in Fig. 5 marks the interface between the two particle clouds. Waves travelled from the cen- 


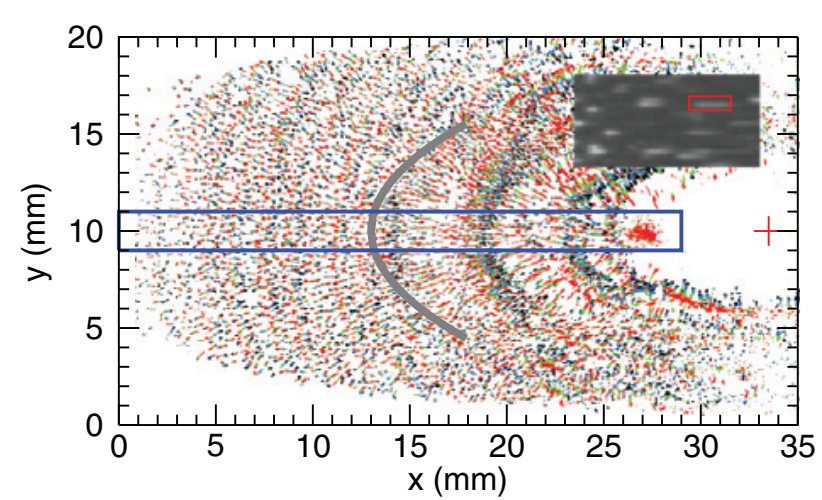

Fig. 5 (color online) Overlay of five consecutive $(\Delta t=0.02 \mathrm{~s})$ experimental snapshots of a binary complex plasma undergoing wave activity. Color codes time and varies from blue to red. The gray curve marks the interface between the areas occupied by small (closer to the void in the right) and large (closer to the cloud edge) microparticles. The blue rectangle encloses the region analyzed in detail. The red cross in the right marks the chamber center. The overlay shows an experimental picture with particle images. Reprinted with permission from Yang et. al, Density waves at the interface of a binary complex plasma, EPL 117 (2017) 25001 [38].

ter of the cloud outwards, across the interface between the two subclouds. In the subcloud composed of larger particles, the waves were self-excited due to two-stream instability with a frequency of $5.4 \pm 0.5 \mathrm{~Hz}$ and a wave length of $1.8 \pm 0.3 \mathrm{~mm}$. The void was slightly oscillating due to heartbeat instability, which lead to waves in the small particle subcloud with a frequency of $3.6 \pm 0.1 \mathrm{~Hz}$ and a wave length of $4.2 \pm 0.3 \mathrm{~mm}$. By studying the dynamics of the wave crests as they travel near and across the interface, it is possible to identify a collision zone between the wave fronts located before the interface, that is, towards the void, and a merger zone behind it. These results can provide a generic picture of wave-wave interaction at an interface [38].

\section{Photophoretic force}

Even though the majority of the particles participate in collective dynamics, sometime individual particles display abnormal behavior. These particles are also termed "crazy" particles because of their erratic movement. Under gravity conditions, they can be levitated above or below a monolayer of microparticles, disturbing the layer [71-75]. In microgravity, they can be spontaneously accelerated and enter the main microparticle cloud, exciting Mach cones if traveling faster than the speed of sound [23,76], or moving almost freely through the bulk of microparticles [77]. The majority of crazy particles move in direction of the laser beam, which is indicative of radiation pressure as driving force. However, it is possible to observe particle motion not correlated with the direction of the laser beam [78,79]. This irregular mo-
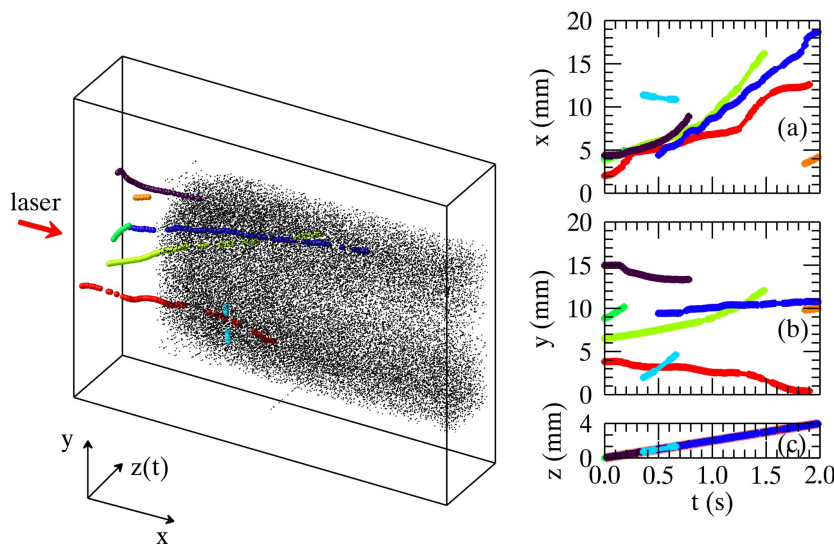

Fig. 6 (color online) Three-dimensional reconstruction (left) of microparticle positions (black dots) and abnormal trajectories (colored lines), and time dependence of $x, y$, and $z$ position of the abnormal particles (right). The microparticle cloud was scanned with a laser sheet in $z$ direction with a speed of $2 \mathrm{~mm} / \mathrm{s}$. The abnormal particles follow the laser scan. From [39].

tion might be caused by an uneven heating of the particle surface and subsequent photophoretic force.

Du et al. [39] studied particles with abnormal trajectories in PK-3 Plus in detail. Again, in the majority of cases, the crazy particles moved in the direction of the laser light, i.e. from left to right. More complicated motion was also present. In particular, particles can become trapped by the laser sheet and, for instance, follow a scan of the laser sheet across the microparticle cloud. Fig. 6 shows such abnormal trajectories of particles observed during a scan. Typically, in the absence of scans, the motion of the abnormal particles was mostly random in the direction transverse to the laser sheet. This suggests that it was driven by photophoretic force [39].

We can not only study the interaction of microscopic projectiles with the microparticle cloud, but also that of macroscopic objects, as we shall see in the following.

\section{Complex plasma - sphere interaction}

In the last experiment of the PK-3 Plus Laboratory, the dispensers were shaken so rigorously that one sieve broke. This caused all microparticles still contained inside to fall into the plasma, as well as $1 \mathrm{~mm}$ diameter metal spheres that were present inside the dispenser to break up any microparticle clumps during shaking [40]. The spheres then interacted with the microparticle cloud. Under microgravity conditions, they do not drop to the ground, which makes it possible to study their interaction with the complex plasma longer and in more details than on ground. In order to impart momentum on the spheres, the cosmonaut who did the experiment, Pavel Vinogradov, manually shoke the experiment container. 


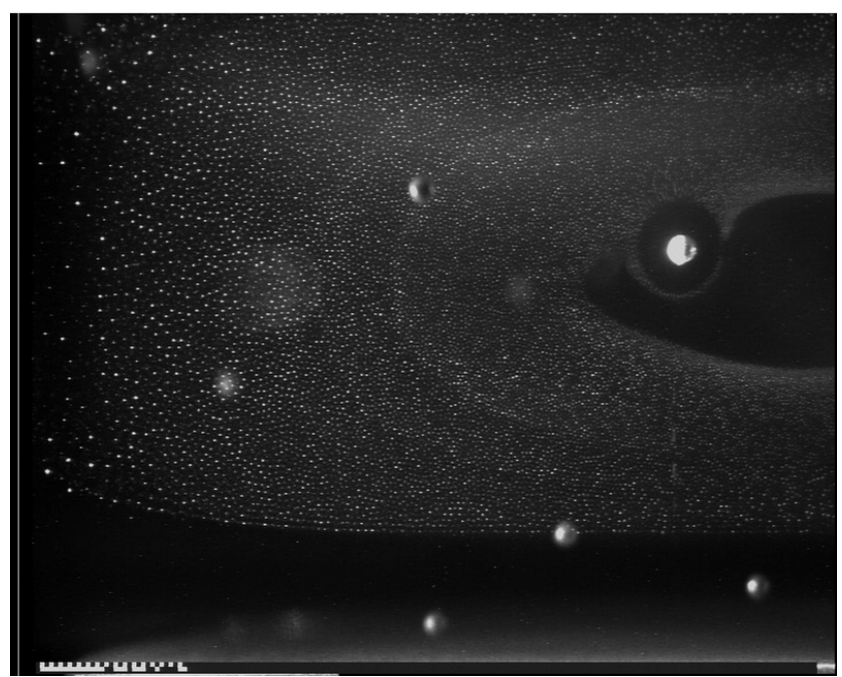

Fig. 7 Experimental snap shot of a complex plasma containing microparticles of sizes $3.4 \mu \mathrm{m}$ and $6.8 \mu \mathrm{m}$, and $1 \mathrm{~mm}$ diameter spheres. When the spheres cross the laser plane, microparticle-free cavities surrounding them are visible, such as in the right of the image. The spheres are visible even when outside of the central part of the laser plane, as they reflect much more light than the microparticles. Field of view: $26 \times 36 \mathrm{~mm}^{2}$.

Schwabe et al. [40] showed that the motion of the metallic spheres was basically free floating during the interaction time with the microparticles, but the microparticles in turn were strongly influenced by the spheres. At small distances, they were strongly repelled, as both microparticles and spheres were charged negatively by the electrons from the plasma. This caused the formation of cavities in the microparticle cloud surrounding the spheres (see Fig. 7).

At intermediate distances from the spheres, the microparticles were attracted to the sphere. This is a consequence of drag of the (positively charged) ions streaming towards the sphere surface. This interplay of long-range attractive and short-range repulsive forces has been found before in a complex plasma interacting with a negatively biased wire [80]. In the present experiment, the enhancement of the ion flux also leads to the excitation of dust acoustic waves when the gas pressure was low enough and the local electric field crossed a threshold [40].

tra found in turbulent microparticle clouds [36]. Last but not least we showed that macroscopic objects immersed in a complex plasma strongly influence the microparticle dynamics [40]. All these examples demonstrate the versatility of the PK-3 Plus Laboratory. In the future, many more interesting results are expected from the successor experiment, PK-4 [31], and the planned Ekoplasma Laboratory [81].

\section{References}

1. C. Colin, Microgravity Sci. Technol. 13, 16 (2002). DOI 10.1007/BF02872067

2. Z. L. Wang, K. S. Gabriel, Z. F. Zhu, Microgravity Sci. Technol. 15, 19 (2004). DOI 10.1007/BF02870961

3. Y. Liu, G. Li, S. Kallio, Microgravity Sci. Technol., 23, 1 (2010). DOI 10.1007/s12217-010-9199-4

4. O. Bozier, B. Veyssière, Microgravity Sci. Technol. 22, 233 (2010). DOI 10.1007/s12217-010-9184-y

5. G.E. Morfill, A.V. Ivlev, Rev. Mod. Phys. 81, 1353 (2009). DOI 10.1103/RevModPhys.81.1353

6. A. Melzer, Phys. Rev. E 90, 053103 (2014). DOI 10.1103/PhysRevE.90.053103

7. R. Kompaneets, G.E. Morfill, A.V. Ivlev, Phys. Rev. E 93, 063201 (2016). DOI 10.1103/PhysRevE.93.063201

8. T.M. Flanagan, J. Goree, Phys. Plasmas 17, 123702 (2010). DOI $10.1063 / 1.3524691$

9. A.P. Nefedov, G.E. Morfill, V.E. Fortov, H.M. Thomas, H. Rothermel, T. Hagl, A.V. Ivlev, M. Zuzic, B.A. Klumov, A.M. Lipaev, V.I. Molotkov, O.F. Petrov, Y.P. Gidzenko, S.K. Krikalev, W. Shepherd, A.I. Ivanov, M. Roth, H. Binnenbruck, J.A. Goree, Y.P. Semenov, New J. Phys. 5, 33 (2003). DOI 10.1088/13672630/5/1/333

10. H. M. Thomas, G. E. Morfill, A. V. Ivlev, A. P. Nefedov, V. E. Fortov, H. Rothermel, M. Rubin-Zuzic, A. M. Lipaev, V. I. Molotkov, O. F. Petrov, Micrograv. Sci. Technol. 16, 317 (2005). DOI 10.1007/BF02945998

11. G.E. Morfill, H.M. Thomas, U. Konopka, H. Rothermel, M. Zuzic, A. Ivlev, J. Goree, Phys. Rev. Lett. 83, 1598 (1999). DOI 10.1103/PhysRevLett.83.1598

12. J. Goree, G.E. Morfill, V.N. Tsytovich, S.V. Vladimirov, Phys Rev. E 59(6), 7055 (1999). DOI 10.1103/PhysRevE.59.7055

13. A.M. Lipaev, S.A. Khrapak, V.I. Molotkov, G.E. Morfill, V.E. Fortov, A. Ivlev, H.M. Thomas, A.G. Khrapak, V.N. Naumkin, A.I. Ivanov, S.E. Tretschev, G.I. Padalka, Phys. Rev. Lett. 98, 265006 (2007). DOI 10.1103/PhysRevLett.98.265006

14. P. Huber, Untersuchung von 3-d plasmakristallen. Ph.D. thesis, Ludwig-Maximilians-Universität München (2011)

15. S. Khrapak, D. Samsonov, G. Morfill, H. Thomas, V. Yaroshenko, H. Rothermel, T. Hagl, V. Fortov, A. Nefedov, V. Molotkov, O. Petrov, A. Lipaev, A. Ivanov, Y. Baturin, Phys. Plasmas 10 1 (2003). DOI 10.1063/1.1525283

16. M. Himpel, T. Bockwoldt, C. Killer, K.O. Menzel, A. Piel, A. Melzer, Phys. Plasmas 21, 033703 (2014). DOI $10.1063 / 1.4868859$

17. K.R. Sütterlin, A. Wysocki, A.V. Ivlev, C. Räth, H.M. Thomas, M. Rubin-Zuzic, W.J. Goedheer, V.E. Fortov, A.M. Lipaev, V.I. Molotkov, O.F. Petrov, G.E. Morfill, H. Löwen, Phys. Rev. Lett. 102, 085003 (2009). DOI 10.1103/PhysRevLett.102.085003

18. C.R. Du, K.R. Sütterlin, K. Jiang, C. Räth, A.V. Ivlev, S. Khrapak, M. Schwabe, H.M. Thomas, V.E. Fortov, A.M. Lipaev, V.I. Molotkov, O.F. Petrov, Y. Malentschenko, F. Yurtschichin, Y. Lonchakov, G.E. Morfill, New J. Phys. 14, 073058 (2012). DOI 10.1088/1367-2630/14/7/073058 
19. Y. Hayashi, Microgravity Sci. Technol. 16, 64 (2005). DOI $10.1007 / \mathrm{bf} 02945948$

20. A.V. Ivlev, G.E. Morfill, H.M. Thomas, C. Räth, G. Joyce, P. Huber, R. Kompaneets, V.E. Fortov, A.M. Lipaev, V.I. Molotkov, T. Reiter, M. Turin, P. Vinogradov, Phys. Rev. Lett. 100, 095003 (2008). DOI 10.1103/PhysRevLett.100.095003

21. K. Takahashi, M. Tonouchi, S. Adachi, H. Totsuji, Int. J. Microgravity Sci. Appl. 31, 18 (2014)

22. C. Dietz, M. Kretschmer, B. Steinmüller, M.H. Thoma, Contrib. Plasma Phys. (2017). DOI 10.1002/ctpp.201700055

23. K. Jiang, V. Nosenko, Y.F. Li, M. Schwabe, U. Konopka, A.V. Ivlev, V.E. Fortov, V.I. Molotkov, A.M. Lipaev, O.F. Petrov, M.V. Turin, H.M. Thomas, G.E. Morfill, EPL 85, 45002 (2009). DOI 10.1209/0295-5075/85/45002

24. D. Caliebe, O. Arp, A. Piel, Phys. Plasmas 18, 073702 (2011). DOI 10.1063/1.3606468

25. D.I. Zhukhovitskii, V.E. Fortov, V.I. Molotkov, A.M. Lipaev, V.N. Naumkin, H.M. Thomas, A.V. Ivlev, M. Schwabe, G.E. Morfill, Phys. Plasmas 22, 023701 (2015)

26. V.E. Fortov, A.P. Nefedov, O.S. Vaulina, A.M. Lipaev, V.I. Molotkov, A.A. Samaryan, V.P. Nikitskii, A.I. Ivanov, S.F. Savin, A.V. Kalmykov, A.Y.S. ev, P.V. Vinogradov, J. Exp. Theo. Phys. 87(6), 1087 (1998). DOI 10.1134/1.558598

27. H.M. Thomas, G.E. Morfill, V.E. Fortov, A.V. Ivlev, V.I. Molotkov, A.M. Lipaev, T. Hagl, H. Rothermel, S.A. Khrapak, R.K. Sütterlin, M. Rubin-Zuzic, O.F. Petrov, V.I. Tokarev, S.K. Krikalev, New J. Phys. 10, 033036 (2008). DOI 10.1088/13672630/10/3/033036

28. V.I. Molotkov, H.M. Thomas, A.M. Lipaev, V.N. Naumkin, A.V. Ivlev, S.A. Khrapak, Int. J. Microgravity Sci. Appl. 32(3), 320302 (2015). DOI 10.15011/jasma.32.320302

29. A.G. Khrapak, V.I. Molotkov, A.M. Lipaev, D.I. Zhukhovitskii, V.N. Naumkin, V.E. Fortov, O.F. Petrov, H.M. Thomas, S.A. Khrapak, P. Huber, A. Ivlev, G. Morfill, Contrib. Plasma Phys. 56, 253 (2016). DOI 10.1002/ctpp.201500102

30. M. H. Thoma, H. Höfner, M. Kretschmer, S. Ratynskaia, G. E. Morfill, A. Usachev, A. Zobnin, O. Petrov, V. Fortov, Microgravity Sci. Technol. 18, 47 (2006). DOI 10.1007/BF02870378

31. M.Y. Pustylnik, M.A. Fink, V. Nosenko, T. Antonova, T. Hagl, H.M. Thomas, A.V. Zobnin, A.M. Lipaev, A.D. Usachev, V.I. Molotkov, O.F. Petrov, V.E. Fortov, C. Rau, C. Deysenroth, S. Albrecht, M. Kretschmer, M.H. Thoma, G.E. Morfill, R. Seurig, A. Stettner, V.A. Alyamovskaya, A. Orr, E. Kufner, E.G. Lavrenko, G.I. Padalka, E.O. Serova, A.M. Samokutyayev, S. Christoforetti, Review of Scientific Instruments 87, 093505 (2016). DOI 10.1063/1.4962696

32. Y. Feng, J. Goree, B. Liu, Rev. Sci. Instrum. 78, 053704 (2007). DOI $10.1063 / 1.2735920$

33. D.I. Zhukhovitskii, V.N. Naumkin, A.I. Khusnulgatin, V.I. Molotkov, A.M. Lipaev, Phys. Rev. E 96, 043204 (2017). DOI 10.1103/PhysRevE.96.043204

34. V.N. Naumkin, D.I. Zhukhovitskii, V.I. Molotkov, A.M. Lipaev, V.E. Fortov, H.M. Thomas, P. Huber, G.E. Morfill, Phys. Rev. E 94, 033204 (2016). DOI 10.1103/PhysRevE.94.033204

35. S. Zhdanov, C.R. Du, M. Schwabe, V. Nosenko, H.M. Thomas, G.E. Morfill, EPL 114, 55002 (2016). DOI 10.1209/02955075/114/55002

36. M. Schwabe, S. Zhdanov, C. Räth, Phys. Rev. E 95, 041201(R) (2017). DOI 10.1103/PhysRevE.95.041201

37. M. Schwabe, S. Zhdanov, C. Räth, IEEE Trans. Plasma Sci. PP, 1 (2017). DOI 10.1109/TPS.2017.2744682

38. L. Yang, M. Schwabe, S. Zhdanov, H.M. Thomas, A.M. Lipaev, V.I. Molotkov, V.E. Fortov, J. Zhang, C.R. Du, EPL 117(2), 25001 (2017). DOI 10.1209/0295-5075/117/25001

39. C.R. Du, V. Nosenko, H.M. Thomas, A. Müller, A.M. Lipaev, V.I. Molotkov, V.E. Fortov, A.V. Ivlev, New J. Phys. 19(7), 073015 (2017). DOI 10.1088/1367-2630/aa724f
40. M. Schwabe, S.K. Zhdanov, T. Hagl, P. Huber, A.M. Lipaev, V.I. Molotkov, V. Naumkin, M. Rubin-Zuzic, P.V. Vinogradov, E. Zähringer, V.E. Fortov, H.M. Thomas, New J. Phys. 19, 103019 (2017). DOI 10.1088/1367-2630/aa868c

41. H. Thomas, G.E. Morfill, V. Demmel, J. Goree, B. Feuerbacher, D. Möhlmann, Phys. Rev. Lett. 73(5), 652 (1994). DOI 10.1103/PhysRevLett.73.652

42. A. Melzer, A. Homann, A. Piel, Phys. Rev. E 53, 27572766 (1996). DOI 10.1103/PhysRevE.53.2757

43. H.M. Thomas, G.E. Morfill, Nature 379, 806 (1996). DOI $10.1038 / 379806 a 0$

44. S.A. Khrapak, B.A. Klumov, P. Huber, V.I. Molotkov, A.M. Lipaev, V.N. Naumkin, A.V. Ivlev, H.M. Thomas, M. Schwabe, G.E. Morfill, O.F. Petrov, V.E. Fortov, Y. Malentschenko, S. Volkov, Phys. Rev. E 85, 066407 (2012). DOI 10.1103/PhysRevE.85.066407

45. B. Steinmüller, C. Dietz, M. Kretschmer, M.H. Thoma, Phys. Plasmas 24, 033705 (2017). DOI 10.1063/1.4977902

46. V.I. Molotkov, V.N. Naumkin, A.M. Lipaev, D.I. Zhukhovitskii, A.D. Usachev, V.E. Fortov, H.M. Thomas, Journal of Physics: Conference Series 927, 012037 (2017). DOI 10.1088/17426596/927/1/012037

47. M. Rubin-Zuzic, G.E. Morfill, H.M. Thomas, A.V. Ivlev, B.A. Klumov, H. Rothermel, W. Bunk, R. Pompl, O. Havnes, A. Fouquét, Nature Physics 2, 181 (2006). DOI 10.1038/nphys242

48. M. Schwabe, M. Rubin-Zuzic, S. Zhdanov, A.V. Ivlev, H.M. Thomas, G.E. Morfill, Phys. Rev. Lett. 102, 255005 (2009). DOI 10.1103/PhysRevLett.102.255005

49. G. Gozadinos, A.V. Ivlev, J.P. Boeuf, New J. Phys. 5, 32 (2003). DOI 10.1088/1367-2630/5/1/332

50. A. Melzer, A. Homann, A. Piel, Phys. Rev. E 53, 2757 (1996). DOI 10.1103/PhysRevE.53.2757; V. A. Schweigert, I. V. Schweigert, A. Melzer, A. Homann, A. Piel. Phys. Rev. E 54, 4155 (1996). DOI 10.1103/PhysRevE.54.4155; G. Joyce, M. Lampe, G. Ganguli, Phys. Rev. Lett. 88, 095006 (2002). DOI 10.1103/PhysRevLett.88.095006; J. D. Williams, E. Thomas, Phys. Plasmas 14, 063702 (2007). DOI 10.1063/1.2741457; R. Fisher, K. Avinash, E. Thomas, R. Merlino, V. Gupta, Phys. Rev. E 88, 031101(R) (2013). DOI 10.1103/physreve.88.031101

51. D. Samsonov, J. Goree, Phys. Rev. E 59(1), 1047 (1999). DOI 10.1103/PhysRevE.59.1047

52. R. Heidemann, L. Couëdel, S. Zhdanov, K. Sütterlin, M. Schwabe, H. Thomas, A. Ivlev, T. Hagl, G. Morfill, V. Fortov, V. Molotkov, O. Petrov, A. Lipaev, V. Tokarev, T. Reiter, P. Vinogradov, Phys. Plasmas 18, 053701 (2011). DOI 10.1063/1.3574905

53. M.Y. Pustylnik, A.V. Ivlev, N. Sadeghi, R. Heidemann, S. Mitic, H.M. Thomas, G.E. Morfill, Phys. Plasmas 19, 103701 (2012). DOI 10.1063/1.4757213

54. R.H. Kraichnan, Phys. Fluids 10, 1417 (1967). DOI $10.1063 / 1.1762301$

55. C.E. Leith, Phys. Fluids 11, 671 (1968). DOI 10.1063/1.1691968

56. N.N. Rao, P.K. Shukla, M.Y. Yu, Planet. Space Sci. 38(4), 543 (1990). DOI 10.1016/0032-0633(90)90147-I

57. A. Barkan, R.L. Merlino, N. D’Angelo, Phys. Plasmas 2(10), 3563 (1995). DOI 10.1063/1.871121

58. P.K. Shukla, M.M. Mamun, Inatroduction to Dusty Plasma Physics (IOP, Bristol, 2002)

59. R.L. Merlino, Phys. Plasmas 16, 124501 (2009). DOI $10.1063 / 1.3271155$

60. R.L. Merlino, J. Plasma Phys. p. 1 (2014). DOI $10.1017 /$ S0022377814000312

61. M. Mikikian, L. Couëdel, M. Cavarroc, Y. Tessier, L. Boufendi, New J. Phys. 9, 268 (2007). DOI 10.1088/1367-2630/9/8/268

62. T. Trottenberg, D. Blick, A. Piel, Phys. Plasmas 13, 042105 (2006). DOI 10.1063/1.2196347 
63. M. Schwabe, S.K. Zhdanov, H.M. Thomas, A.V. Ivlev, M. RubinZuzic, G.E. Morfill, V.I. Molotkov, A.M. Lipaev, V.E. Fortov, T. Reiter, New J. Phys. 10, 033037 (2008). DOI 10.1088/13672630/10/3/033037

64. E. Thomas, Phys. Plasmas 17, 043701 (2010). DOI 10.1063/1.3369846

65. J.D. Williams, Phys. Plasmas 19, 113701 (2012). DOI 10.1063/1.4766813

66. M. Schwabe, M. Rubin-Zuzic, S. Zhdanov, H.M. Thomas, G.E. Morfill, Phys. Rev. Lett. 99(9), 095002 (2007). DOI 10.1103/PhysRevLett.99.095002

67. C.T. Liao, L.W. Teng, C.Y. Tsai, C.W. Io, L. Ivanov, Phys. Rev. Lett. 100, 185004 (2008). DOI 10.1103/PhysRevLett.100.185004

68. L.J. Hou, A. Piel, Phys. Plasmas 15, 073707 (2008). DOI 10.1063/1.2956991

69. M. Himpel, C. Killer, B. Buttenschön, A. Melzer, Phys. Plasmas 19, 123704 (2012). DOI 10.1063/1.4771687

70. A.V. Ivlev, S.K. Zhdanov, H.M. Thomas, G.E. Morfill, EPL 85, 45001 (2009). DOI 10.1209/0295-5075/85/45001

71. D. Samsonov, J. Goree, Z.W. Ma, A. Bhattacharjee, H.M. Thomas, G.E. Morfill, Phys. Rev. Lett. 83(18), 3649 (1999). DOI 10.1103/PhysRevLett.83.3649

72. D.H.E. Dubin, Phys. Plasmas 7, 3895 (2000). DOI $10.1063 / 1.1308078$

73. V.A. Schweigert, I.V. Schweigert, V. Nosenko, J. Goree, Phys. Plasmas 9(11), 4465 (2002). DOI 10.1063/1.1512656

74. O. Havnes, T.W. Hartquist, A. Brattli, G.M.W. Kroesen, G. Morfill, Physical Review E 65(4) (2002). DOI 10.1103/PhysRevE.65.045403

75. C.R. Du, V. Nosenko, S. Zhdanov, H.M. Thomas, G.E. Morfill, EPL 99, 55001 (2012). DOI 10.1209/0295-5075/99/55001

76. M. Schwabe, K. Jiang, S. Zhdanov, T. Hagl, P. Huber, A.V. Ivlev, A.M. Lipaev, V.I. Molotkov, V.N. Naumkin, K.R. Sütterlin, H.M. Thomas, V.E. Fortov, G.E. Morfill, A. Skvortsov, S. Volkov, EPL 96, 55001 (2011). DOI 10.1209/0295-5075/96/55001

77. D.I. Zhukhovitskii, V.E. Fortov, V.I. Molotkov, A.M. Lipaev, V.N. Naumkin, H.M. Thomas, A.V. Ivlev, M. Schwabe, G.E. Morfill, Phys. Rev. E 86, 016401 (2012). DOI 10.1103/PhysRevE.86.016401

78. V. Nosenko, A.V. Ivlev, G.E. Morfill, Phys. Plasmas 17, 123705 (2010). DOI 10.1063/1.3525254

79. M. Küpper, C. de Beule, G. Wurm, L.S. Matthews, J.B. Kimery, T.W. Hyde, J. Aerosol Sci. 76, 126 (2014). DOI 10.1016/j.jaerosci.2014.06.008

80. D. Samsonov, A.V. Ivlev, G.E. Morfill, J. Goree, Phys. Rev. E 63, 025401(R) (2001). DOI 10.1103/PhysRevE.63.025401

81. C.A. Knapek, P. Huber, D.P. Mohr, E. Zaehringer, V.I. Molotkov, A.M. Lipaev, V. Naumkin, U. Konopka, H.M. Thomas, V.E. Fortov, in AIP Conference Proceedings (Author(s), 2018). DOI $10.1063 / 1.5020392$ 